\title{
The role of leukotrienes in the pathogenesis of systemic sclerosis
}

\author{
Sylwia Chwieśko-Minarowska ${ }^{1}$, Krzysztof Kowal' ${ }^{2}$, \\ Marek Bielecki ${ }^{3}$, Otylia Kowal-Bielecka ${ }^{4}$
}

${ }^{1}$ Department of Rehabilitation, Medical University of Bialystok, Poland

${ }^{2}$ Department of Allergology and Internal Medicine, Medical University of Bialystok, Poland

${ }^{3}$ Department of Orthopedics and Traumatology, Medical University of Bialystok, Poland

${ }^{4}$ Department of Rheumatology and Internal Medicine, Medical University of Bialystok, Poland

\begin{abstract}
Systemic sclerosis (SSc, scleroderma) is an autoimmune disease characterized by widespread vascular injury and progressive fibrosis of the skin and internal organs. SSc-related involvement of the lungs, heart, kidneys and/or the gastrointestinal system accounts for the increased mortality of scleroderma patients. Despite the progress which has recently been made in this field, the treatment of SSc is still unsatisfactory due to the low efficacy and/or high toxicity of available therapies. Leukotrienes are a family of lipid mediators synthesized from arachidonic acid in a process mediated by 5-lipoxygenase; they include leukotriene B4 and a group of cysteinyl leukotrienes: C4, D4, and E4. Leukotrienes play an important role in the regulation of all the processes vital to the pathogenesis of SSc, namely inflammation, vascular function and connective tissue remodeling. The available data suggests that an excessive synthesis of leukotrienes may contribute to the development and progression of SSc. Accordingly, blockade of leukotriene pathways appears to be a new, promising target for the treatment of SSc. (Folia Histochemica et Cytobiologica 2012, Vol. 50, No. 2, 180-185)
\end{abstract}

Key words: leukotrienes, scleroderma, SSc-related insterstitial lung disease

\section{Introduction}

Systemic sclerosis (SSc, scleroderma) is an autoimmune disease involving the skin, musculoskeletal system, lungs, heart, gastrointestinal tract and kidneys. The involvement of internal organs results in increased mortality of patients with SSc. In particular, pulmonary complications such as SSc-related interstitial lung disease (SSc-ILD, pulmonary fibrosis) and pulmonary hypertension are currently the leading causes of disease-related deaths $[1,2]$.

There are no universal disease-modifying treatments available in SSc. Despite the progress that has

Correspondence address: $O$. Kowal-Bielecka, Department of Rheumatology and Internal Medicine, Medical University of Bialystok,

M. Sklodowskiej-Curie Str. 24A, 15-276 Bialystok, Poland; tel: + 488574684 82, fax: + 48857468606 ;

e-mail: otylia@umb.edu.pl recently been made in the management of specific SSc-related organ involvement, the efficacy of SSc treatment remains unsatisfactory [3]. Moreover, the toxicity of available drugs is still significant, meaning that new potent therapeutic targets are being sought $[4,5]$.

The etiology of SSc is unknown and its pathogenesis not fully understood. Although excessive fibrosis is the most characteristic pathological feature of SSc, it is considered to be a late and irreversible stage of the disease. In addition to fibrosis, vascular injury and tissue inflammation are considered to be important contributors to SSc pathology.

\section{Leukotrienes - definition and synthesis}

Leukotrienes represent a group of lipid mediators synthesized from arachidonic acid in a process mediated by 5 -lipoxygenase (5-LOX). The group of leukotrienes includes leukotriene A4 (LTA4), a short- 


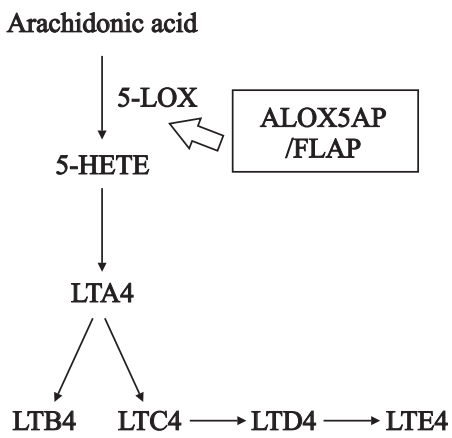

Figure 1. Leukotriene synthesis pathway

5-LOX - 5-lipoxygenase; 5-HETE - 5-hydroperoxyeicosatetraenoic acid; ALOX5AP/FLAP - 5-lipoxygenase activating protein; LTA4 - leukotriene A4; LTB4 - leukotriene B4; LTC4 - leukotriene C4; LTD4 - leukotriene D4; LTE4 - leukotriene E4

-living intermediate product of the leukotriene synthesis pathway, leukotriene B4 (LTB4), and the group of cysteinyl leukotrienes: C4 (LTC4), D4 (LTD4), and E4 (LTE4).

Cysteinyl leukotrienes belong to the group of proinflammatory mediators and are referred to as slow reacting substances of anaphylaxis [6,7]. Leukotrienes were discovered in animal lungs during provocation challenges with snake's venom, the main enzymatic component of which is phospholipase A2. Stimulation with immunological, allergic, or inflammatory factors initiates the synthesis of leukotrienes from phospholipids as a result of the release of intracellular $\mathrm{Ca}^{2+}$ ions that subsequently induce translocation of cytosolic phospholipase A2 alpha and 5-LOX into the endosomal structures of the cell [8].

The synthesis of leukotrienes requires the presence of 5-lipoxygenase activating protein (ALOX5AP/ /FLAP). The ALOX5AP/FLAP protein is thought to participate in selective translocation of arachidonic acid to 5-LOX, which catalyzes its oxygenation to 5-hydroperoxyeicosatetraenoic acid (5-HpETE) and a further dehydration of the latter to the unstable leukotriene LTA4. As a result of hydrolysis, LTA4 is transformed into pro-inflammatory LTB4, a chemotactic factor and leukocyte activator - Figure $1[8,9]$.

In another metabolic pathway, LTA4 is bound to glutathione by LTC4 synthase leading to the generation of leukotrienes containing a cysteinyl group: LTC4, LTD4, and LTE4 [8].

Leukocytes constitute the principal source of leukotrienes and the profile of the latter depends on the type of white blood cell. Neutrophils produce mainly LTB4, eosinophils and basophils synthesize cysteinyl leukotrienes, while monocytes/macrophages can generate both groups of leukotrienes. Additionally, other cells (fibroblasts, epithelial cells, blood platelets) have been shown to be able to synthesize leukotrienes, but to a lesser extent [6].

\section{Biological function of leukotrienes}

Leukotrienes act via the activation of cell-surface protein G-bound receptors. Depending on the type of $\mathrm{G}$ protein, receptors transmit the signal by changing the intracellular cAMP, calcium, or diacylglycerol levels. Leukotriene LTB4 acts via BLT1 and BLT2 receptors, while cysteinyl leukotrienes activate CysLT1 and CysLT2 receptors. Leukotrienes are considered to be pro-inflammatory mediators [reviewed in more detail in refs. 8 and 9]. Leukotriene LTB4 is one of the strongest neutrophil chemoattractants and activators. Through the activation of NF $\kappa$ B factor and AP1, LTB4 stimulates the synthesis of various pro-inflammatory cytokines, including tumor necrosis factor alpha $(\mathrm{TNF} \alpha)$, interleukin 6 (IL-6), interleukin 8 (IL-8), and fibroblast growth factor (FGF) [8]. Leukotriene LTB4 activates monocytes/macrophages to synthesize and release mediators responsible for the aggregation of other immune cells, i.e. T lymphocytes, in the area involved in the inflammatory process. Activated T lymphocytes can, in turn, induce enhanced expression of ALOX5AP/FLAP protein in macrophages, leading to the intensified synthesis of leukotriene LTB4 [9]. These processes produce a positive feedback loop which plays a role in amplification of the inflammatory response. Novel anti-leukotriene therapies may reveal their anti-inflammatory properties by interrupting this 'vicious circle'.

Leukotrienes have been shown to modulate the function of blood vessels [9-11]. Cysteinyl leukotrienes powerfully contract bronchial and vascular smooth muscles, increase the permeability of blood vessels, and activate endothelial cells $[9,11]$. Furthermore, leukotrienes are involved in the proliferation and migration of vascular smooth muscle cells $[9,10]$. It has recently been shown that the role of leukotrienes in vascular biology is more complex, and vasomotor response to leukotrienes depends on the functional status of vascular endothelium as well as on the expression of a given subtype of leukotriene receptor. Accordingly, activation of CysLT2 receptors on endothelial cells can lead to the synthesis of nitric oxide (NO), a powerful vasodilatory and anti-proliferative mediator, while stimulation of endothelial CysLT1 receptor may in contrast result in the release of contractile factors [reviewed in ref. 9]. In some pathologic conditions, LTB4 can affect endothelial cell biology in yet another way. The complex of LTB4 with proteins has been shown to be responsible for cytotoxic effects directed against the endothelial cells in SSc patients [12]. 
Leukotrienes have also been shown to be involved in the regulation of fibroblast function and connective tissue remodeling [13]. Leukotriene LTB4 stimulates the migration of fibroblasts. Cysteinyl leukotrienes induce synthesis of collagen, differentiation of fibroblasts into myofibroblasts, and stimulate epithelial cells to synthesize transforming growth factor beta (TGF-beta), which plays a pivotal role in the processes of fibrosis [13]. Moreover, leukotrienes may contribute to the regulation of synthesis, secretion, and activation of matrix metalloproteinases (MMPs) which play a key role in the remodeling of connective tissue [14]. It has been shown that 5-LOX and MMPs are co-expressed in the atherosclerotic lesions of the carotid artery [15].

\section{Leukotrienes and systemic sclerosis}

Leukotrienes play an important role in the regulation of all the processes vital to the development of SSc, namely inflammatory response, blood vessel function and connective tissue synthesis. Accordingly, both the results of human studies and the experimental data suggest that an impaired balance between various classes of eicosanoids, and particularly excessive synthesis of leukotrienes, may be of key importance to the SSc pathogenesis.

It has been shown that 5-LOX, a key enzyme in leukotriene biosynthesis, is overexpressed in the skin of patients with SSc compared to healthy controls [16]. Expression of 5-LOX has been mainly found in mononuclear cells within perivascular inflammatory infiltrates, but also in fibroblast-like cells throughout the skin. In vitro studies have revealed that skin fibroblast from both SSc patients and healthy subjects express 5-LOX and synthesize leukotrienes, with fibroblast from SSc patients producing significantly greater amounts of LTB4 compared to cells derived from healthy subjects. Despite a significantly higher synthesis of LTB4, no significant difference in the level of expression of 5-LOX between SSc fibroblasts and cells derived from healthy controls has been revealed. Recently, however Xiao et al. showed that in cultured fibroblasts from early (of less than two years' duration) diffuse SSc patients, the expression of 5-LOX was significantly greater than in normal human dermal fibroblasts [17]. The discrepancies between the two above-mentioned studies regarding expression of 5-LOX in SSc fibroblasts could potentially be explained by the heterogeneity of fibroblasts derived from patients with SSc, with cells taken from early diffuse SSc being most active [18]. Indeed, the relevance of overexpression of 5-LOX in pro-fibrotic activities of SSc fibroblast seems to be further confirmed by the results of Xiao and al. who showed that inhibi- tion of 5-LOX with MK886, a specific 5-LOX inhibitor, was associated with decreased expression of collagen types I and III as well as two cytokines considered to be of key importance for SSc pathogenesis: transforming growth factor beta1 (TGFbeta1) and connective tissue growth factor (CTGF) [17].

Human studies indicate that increased synthesis of leukotrienes (activation of the leukotriene pathway) might also play an important role in the development of scleroderma-related interstitial lung disease (SSc-ILD) [reviewed in more detail in ref. 19]. High concentrations of LTB4 and LTE4 were found in the lungs of patients with SSc-ILD compared to SSc patients without SSc-ILD and healthy controls [20, 21]. Concentrations of LTB4 and LTE4 had a significantly positive correlation with each other and with the fraction of inflammatory cells in bronchoalveolar lavage fluid (BALF). The concentration of leukotriene LTB4 correlated positively with the percentage of neutrophils, which is considered a marker of local inflammation in the lower respiratory tract and a poor prognostic factor [20, 22]. Moreover, concentrations of both LTB4 and LTE4 in the BALF negatively correlated with FVC [20]. These findings are in agreement with the study of Wilborn et al. who showed that tissue homogenates obtained from patients with idiopathic pulmonary fibrosis (IPF), a prototypic fibrotic lung disease, contained 15 -fold higher concentrations of LTB4, and five-fold higher levels of LTC4, compared to control samples of nonfibrotic lung tissue [23]. The concentration of LTB4 in the homogenates of fibrotic lungs strongly correlated with histopathological indices of inflammation and fibrosis. Moreover, elevated leukotriene concentrations have also been observed in lung fibrosis associated with pigeon breeder's lung [24].

Recently, Tufvesson et al. measured the concentration of leukotrienes in exhaled breath condensates of 22 patients with SSc, including 12 patients with SScILD as defined by the presence of traction bronchiectasis within areas of ground-glass opacities and/ /or reticulation in high resolution computed tomography (HRCT) of the lungs [25]. They showed that concentration of cysteinyl leukotrienes is significantly higher in exhaled breath condensates of SSc patients compared to healthy controls. Although the concentration of LTB4 significantly correlated with the concentration of cysteinyl leukotrienes, the difference in LTB4 levels was not significant between SSc patients and the control group. No association was documented in this group between the leukotriene concentrations and radiological findings of HRCT or functional pulmonary tests [25]. There is no ready explanation for the discrepancies between measurements of 
LTB4 in BALF and exhaled breath condensates; however, it could be due to the limited numbers of patients with SSc-ILD in the study by Tufvesson and/or the influence of treatment which could potentially alter leukotriene metabolism. Indeed, in the study by Tufvesson several patients received non-steroidal antiinflammatory drugs or steroids. These observations are of particular interest in view of our previous results showing that immunosuppressive treatment of SSc-ILD resulted in a significant decrease in the concentration of LTB4, but had no effect on LTE4, in the lungs of patients with SSc-ILD [20].

The exact source of increased synthesis of leukotrienes in the lungs of patients with SSc-ILD has not been identified. Immunohistochemical studies in idiopathic pulmonary fibrosis have shown that 5-LOX is expressed mainly in alveolar macrophages in both patients with pulmonary fibrosis and the control samples of nonfibrotic lung tissue from patients undergoing resectional surgery for bronchogenic carcinoma [23]. In vitro, alveolar macrophages from patients with idiopathic pulmonary fibrosis synthesized significantly greater amounts of LTB4 and LTC4 compared to cells obtained from normal subjects [23]. It can also be supposed that neutrophils, which are known to be increased in the lungs of patients with pulmonary fibrosis and are able to synthesize LTB4, could be an additional source of leukotrienes in the lungs of patients with SSc-ILD. Studies performed in SSc revealed that neutrophils and mononuclear cells from the peripheral blood of SSc patients synthesize higher amounts of leukotrienes than cells obtained from healthy individuals $[26,27]$. In SSc patients, higher levels of LTB4 in the peripheral blood mononuclear cells (PBMC) cultures were associated with more severe disease as indicated by the presence of diffuse skin involvement, restrictive SSc-ILD, pulmonary hypertension, and greater vascular injury in capillaroscopy [28]. Moreover, higher concentrations of leukotrienes in PBMC cultures were associated with subsequent progression of the disease.

Recent studies indicate that leukotrienes may promote fibrosis and contribute to vascular diseases by the activation of platelets, which are a rich source of growth factors. Thrombocytes contain cysteine leukotriene receptors, and platelet activation markers have been demonstrated in BALF of SSc patients [29, 30].

Arterial pulmonary hypertension is another severe complication of SSc and one of the major causes of mortality in SSc [1, 2]. Histologically, pulmonary arterial hypertension is characterized by the proliferation of vascular tunica intima, hypertrophy of tunica media, and fibrosis of pulmonary vessels. No studies investigating leukotriene pathways specifically in SSc- -related pulmonary arterial hypertension have been identified so far. However, Wright et al. reported that 5-LOX and ALOX5AP/FLAP are overexpressed in the lungs of patients with primary pulmonary hypertension, indicating that the leukotriene pathway might be involved in the pathogenesis of this disease. In patients with idiopathic arterial hypertension, the number of 5-LOX positive macrophages was higher and the intensity of staining for 5-LOX and ALOX5AP/FLAP in small pulmonary arteries was greater compared to normal subjects and patients with intestinal lung disease [31].

The results of experiments using animal models further support the significant role of leukotrienes in the pathogenesis of SSc-related organ complications. Moreover, the results of these experiments indicate that inhibition of leukotriene pathways might be of potential therapeutic benefit in the treatment of the most severe SSc-related organ involvement such as SSc-ILD and/or pulmonary hypertension.

It has been shown that the concentration of leukotrienes is increased in the lungs of mice with bleomycine-induced lung fibrosis [32]. It has also been shown that mice deficient in 5-LOX, a key enzyme in leukotriene biosynthesis, were protected against bleomycine-induced lung fibrosis compared to wild mice showing normal expression of 5-LOX [32]. A protective effect was also observed after the administration of a 5-LOX inhibitor (zileuton) [33]. Moreover, it has been shown the LTB4 receptor antagonist diminishes the development of bleomycin-induced pulmonary fibrosis in mice by decreasing inflammation, hydroxyproline content and expression of TGF-beta, IL-6, and IL-13 in the lungs [34].

The present findings concerning receptor blockade of the cysteinyl leukotrienes pathway in the pathogenesis of lung fibrosis are inconsistent. Beller et al. have shown that mice lacking CysLT2 receptor were protected against bleomycin-induced pulmonary fibrosis, while genetic disruption of CysLT1 receptor led to worse fibrotic response in the lungs of animals challenged with bleomycin $[35,36]$. On the other hand, Izumo et al. showed that montelukast, which is a CysLT1 receptor antagonist, inhibits the inflammatory process and development of bleomycin-induced pulmonary fibrosis in mice, and these effects may be associated with a decrease in TGF-beta expression [37]. Studies in asthma have also suggested an important role for the CysLT1 receptor in the development of airway remodeling, which is characterized by proliferation of the bronchial smooth muscles and deposition of extracellular matrix proteins in the bronchial wall. It has been shown that administration of CysLT1 receptor antagonist inhibited new, and re- 
versed existing, bronchial tree remodeling in a mouse model of chronic allergic asthma [38]. Moreover, antagonists of CysLT1 receptor reduced myofibroblast deposition in the airway wall of patients subjected to allergic provocation [39].

There is growing evidence of the role of leukotrienes in the pathogenesis of different types of vascular diseases associated with arterial wall hypertrophy and ischemia-related injury, which are also important elements of SSc-related pathology. It has been shown that overexpression of 5-LOX markedly accelerated the progression of pulmonary hypertension in rats treated with monocrotaline [40]. The administration of 5-LOX inhibitors, zileuton or MK-886, prevented the development of pulmonary hypertension. It has also been shown that inhibition of FLAP reduced pulmonary vascular reactivity in vitro and the development of pulmonary hypertension in hypoxic rats [41]. Moreover, the antagonists of CysLT1 receptor were found to inhibit the hyperplasia of tunica intima resulting from vascular injury in various animal models [10].

The role of leukotrienes in the development of ischemia-related injury has been investigated quite extensively, although the results of these studies gave inconsistent results. Inhibition of leukotriene synthesis has been shown to improve coronary blood flow in isolated rabbit hearts and to diminish myocardial injury in in vivo models of cardiac infarct in rabbits and dogs $[42,43]$. Accordingly, cysteinyl leukotriene receptor antagonists reduced ischemia/reperfusionrelated injury of the heart in ex vivo and in vivo animal models [44, 45] and attenuated the brain injury resulting from both transient and permanent occlusion of cerebral arteries in rats $[46,47]$. However, other studies did not reveal protective effects of the cysteinyl leukotriene or LTB4 receptors antagonists or inhibition of leukotriene synthesis on myocardial infarction area in dogs or rats [48-51]. It cannot be excluded that the differences in the above studies might be, at least in part, due to different methods/compounds used to block leukotriene pathways. A recent study of transgenic mice overexpressing human CysLT2 receptor in the vascular endothelium revealed that activation of that receptor is associated with a higher expression of inflammatory genes, a larger area of myocardial infarction, and greater dysfunction of the heart, indicating that endothelial CysLT2 receptor is involved in the development of ischemia/ /reperfusion injury [52].

In summary, all the studies reviewed so far indicate that leukotrienes are involved in the pathogenesis of SSc through perpetuation of inflammatory processes, direct or indirect activation of fibroblast, and contribution to vascular injury and remodeling. Increased expression of 5-LOX and/or FLAP, as well as enhanced synthesis of leukotrienes, have been observed in SSc and other conditions of similar pathogenesis (e.g. primary pulmonary hypertension and lung fibrosis), further supporting a key role of the leukotriene pathway in the development of SSc-related organ injury. Moreover, experimental data suggests that inhibition of leukotriene pathways might have a beneficial effect in SSc. This seems of particular interest because an inhibitor of leukotriene synthesis (zileuton) or CysLT receptor antagonists (e.g. montelukast, zafirlukast) have been used for the treatment of asthma and other allergic conditions. Moreover, new anti-leukotriene therapies including LTB receptor antagonists and analogs of lipoxins, natural leukotriene antagonists, are under development.

\section{Acknowledgements}

The work of Drs Sylwia Chwiesko-Minarowska, Krzysztof Kowal and Otylia Kowal-Bielecka was supported by research grants from the Polish State Committee for Scientific Research/Polish National Science Center (grants no N N401 097636 and/or N401 597440).

\section{References}

1. Steen VD, Medsger TA. Changes in causes of death in systemic sclerosis, 1972-2002. Ann Rheum Dis. 2007;66:940-944.

2. Tyndall A, Bannert B, Vonk M et al. Causes and risk factors for death in systemic sclerosis: a study from the EULAR Scleroderma Trials and Research (EUSTAR) database. Ann Rheum Dis. 2010;69:1809-1815.

3. Kowal-Bielecka O, Veale DJ. DMARDs in systemic sclerosis: Do they exist? In: Distler O (ed.). Scleroderma-modern aspects of pathogenesis, diagnosis and therapy. Unimed Bremen 2009;89 O. Distler 95.

4. Distler J, Kowal-Bielecka O, Gay S, Distler O. Future therapies: Novel therapeutic regimens and molecular targets. In: Scleroderma-modern aspects of pathogenesis, diagnosis and therapy. Uni-MedVerlag AG, Bremen-London-Boston 2009;97-104.

5. Kowal-Bielecka O, Landewé R, Avouac J et al. EUSTAR Co-Authors. EULAR recommendations for the treatment of systemic sclerosis: a report from the EULAR Scleroderma Trials and Research group (EUSTAR). Ann Rheum Dis. 2009;68:620-628.

6. Peters-Golden M, Brock TG. 5-lipoxygenase and FLAP. Prostaglandins Leukot Essent Fatty Acids. 2003;69:99-109.

7. Evans JF, Ferguson AD, Mosley RT et al. What's all the FLAP about?: 5-lipoxygenase-activating protein inhibitors for inflammatory diseases. Trends Pharmacol Sci. 2008;29:72-78.

8. Peters-Golden M, Canetti C, Mancuso P et al. Leukotrienes: underappreciated mediators of innate immune responses. J Immunol. 2004;173:589-594.

9. Back M. Leukotriene receptors: crucial components in vascular inflammation. Sci World J. 2007;7:1422-1439.

10. Kaetsu Y, Yamamoto Y, Sugihara S et al. Role of cysteinyl leukotrienes in the proliferation and the migration of murine vascular smooth muscle cells in vivo and in vitro. Cardiovasc Res. 2007;76:160-166.

11. Uzonyi B, Lotzer K, Jahn S et al. Cysteinyl leukotriene 2 receptor and protease-activated receptor 1 activate strongly 
correlated early genes in human endothelial cells. Proc Natl Acad Sci USA. 2006;18;103:6326-6331.

12. Deicher HR, Drenk F, Hoffamn G. Identification of an LtB4-protein complex as evidence of endothelial cell cytotoxic activity in progressive systemic sclerosis. Z Rheumatol. 1987;46:196-197.

13. Huang $\mathrm{H}$, Zeng $\mathrm{Z}$, Li J et al. Variants of arachidonate 5-lipoxygenase-activating protein (ALOX5AP) gene and risk of coronary heart disease: a meta-analysis. Arch Med Res. 2010:41:634-641.

14. Ichiyama T, Kajimoto M, Hasegawa M et al. Cysteinyl leukotrienes enhance tumour necrosis factor-alpha-induced matrix metalloproteinase-9 in human monocytes/macrophages. Clin Exp Allergy. 2007;37:608-614.

15. Cipollone F, Mezzetti A, Fazia ML et al. Association between 5-lipoxygenase expression and plaque instability in humans. Arterioscler Thromb Vasc Biol. 2005;25:1665-1670.

16. Kowal-Bielecka O, Distler O, Neidhart M et al. Evidence of 5-lipoxygenase overexpression in the skin of patients with systemic sclerosis. Arthritis Rheum. 2001;44:1865-1875.

17. Xiao R, Yoshida N, Higashi Y et al. Retinoic acids exhibit antifibrotic activity through the inhibition of 5-lipoxygenase expression in scleroderma fibroblasts. J Dermatol. 2011;38:345-353.

18. Krieg T, Perlish JS, Fleischmajer R, Braun-Falco O. Collagen synthesis in scleroderma: selection of fibroblast populations during subcultures. Arch Dermatol Res. 1985;277:373-376.

19. Kowal-Bielecka O, Kowal K, Distler O et al. Mechanisms of disease: leukotrienes and lipoxins in scleroderma lung disease-insights and potential therapeutic implications. Nat Clin Pract Rheumatol. 2007:3:43-51.

20. Kowal-Bielecka O, Distler O, Kowal K et al. Elevated levels of leukotriene B4 and leukotriene E4 in bronchoalveolar lavage fluid from patients with scleroderma lung disease. $\mathrm{Ar}$ thritis Rheum. 2003;48:1639-1646.

21. Kowal-Bielecka O, Kowal K, Distler O et al. Cyclooxygenase- and lipoxygenase-derived eicosanoids in bronchoalveolar lavage fluid from patients with scleroderma lung disease. $\mathrm{Ar}$ thrithis Rheum. 2005;52:3783-3791.

22. Kowal-Bielecka O, Kowal K, Highland KB, Silver RM. Bronchoalveolar lavage fluid in scleroderma interstitial lung disease: technical aspects and clinical correlations: review of the literature. Semin Arthritis Rheum. 2010;40:73-88.

23. Wilborn J, Bailie M, Coffey M. Constitutive activation of 5-lipoxygenase in the lungs of patients with idiopathic pulmonary fibrosis. J Clin Invest. 1996;97:1827-1836.

24. Selman M, Barquín N, Sansores R. Increased levels of leukotriene $\mathrm{C} 4$ in bronchoalveolar lavage from patients with pigeon breeder's disease. Arch Invest Med. 1988;19:127-131.

25. Tufvesson E, Bozovic G, Hesselstrand G et al. Increased cysteinyl-leukotrienes and 8-isoprostane in exhaled breath condensate from systemic sclerosis patients. Rheumatology. 2010;49:2322-2326

26. Lau CS, O'Dowd A, Belch JJ. White blood cell activation in Raynaud's phenomenon of systemic sclerosis and vibration induced white finger syndrome. Ann Rheum Dis. 1992;51:249-252.

27. Łapińska A, Kowal K, Chwiećko J et al. Eicosanoid production in the peripheral blood mononuclear cells (PBMC) from patients with systemic sclerosis. Joint, bone, spine. 2008; 75:246-247.

28. Lapinska A, Bielecki M, Distler O et al. Increased synthesis of leukotrienes by peripheral blood mononuclear cells is associated with more severe disease and worse prognosis in patients with systemic sclerosis. Abstract accepted for the Annual Congress of the European League against Rheumatism 2012.

29. Hasegawa S, Ichiyama T, Hashimoto K et al. Functional expression of cysteinyl leukotriene receptors on human platelets. Platelets. 2010;21:253-259.

30. Kowal-Bielecka O, Kowal K, Lewszuk A, Bodzenta-Lukaszyk A, Walecki J, Sierakowski S. Beta-thromboglobulin and platelet factor-4 in bronchoalveolar lavage fluid of patients with systemic sclerosis. Annals of the Rheumatic Diseases. 2005:64:484-486.

31. Wright L, Tuder RM, Wang J et al. 5-Lipoxygenase and 5-lipoxygenase activating protein (FLAP) immunoreactivity in lungs from patients with primary pulmonary hypertension. Am J Respir Crit Care Med. 1998;157:219-229.

32. Peters-Golden M, Bailie M, Marshall T et al. Protection from pulmonary fibrosis in leukotriene-deficient mice. Am J Respir Crit Care Med. 2002;165:229-235.

33. Failla M, Genovese T, Mazzon E et al. Pharmacological inhibition of leukotrienes in an animal model of bleomycin-induced acute lung injury. Respir Res. 2006;7:137.

34. Izumo T, Kondo M, Nagai A. Effects of a leukotriene B4 receptor antagonist on bleomycin-induced pulmonary fibrosis. Eur Respir J. 2009;34:1444-1451.

35. Beller TC, Friend DS, Maekawa A et al. Cysteinyl leukotriene 1 receptor controls the severity of chronic pulmonary inflammation and fibrosis. Proc Natl Acad Sci USA. 2004; 101:3047-3052.

36. Beller TC, Maekawa A, Friend DS et al. Targeted gene disruption reveals the role of the cysteinyl leukotriene 2 receptor in increased vascular permeability and in bleomycin-induced pulmonary fibrosis in mice. J Biol Chem. 2004; 279:46129-46134.

37. Izumo T, Kondo M, Nagai A. Cysteinyl-leukotriene 1 receptor antagonist attenuates bleomycin-induced pulmonary fibrosis in mice. Life Sci. 2007;80:1882-1886.

38. Henderson WR Jr, Tang LO, Chu SJ. A role for cysteinyl leukotrienes in airway remodeling in a mouse asthma model. Am J Respir Crit Care Med. 2002;1;165:108-116.

39. Kelly MM, Chakir J, Vethanayagam D. Montelukast treatment attenuates the increase in myofibroblasts following lowdose allergen challenge. Chest. 2006;130:741-753.

40. Jones JE, Walker JL, Song Y et al. Effect of 5-lipoxygenase on the development of pulmonary hypertension in rats. $\mathrm{Am}$ J Physiol Heart Circ Physiol. 2004;286:H1775-1784.

41. Voelkel NF, Tuder RM, Wade K et al. Inhibition of 5-lipoxygenase-activating protein (FLAP) reduces pulmonary vascular reactivity and pulmonary hypertension in hypoxic rats. J Clin Invest. 1996;97:2491-2498.

42. Rossoni G, Sala A, Berti F et al. Myocardial protection by the leukotriene synthesis inhibitor BAY X1005: importance of transcellular biosynthesis of cysteinyl-leukotrienes.J Pharmacol Exp Ther. 1996;276:335-341.

43. Mullane K, Hatala MA, Kraemer R et al. Myocardial salvage induced by REV-5901: an inhibitor and antagonist of the leukotrienes. J Cardiovasc Pharmacol. 1987;10:398-406.

44. Lee CC, Appleyard RF, Byrne JG et al. Leukotrienes D4 and E4 produced in myocardium impair coronary flow and ventricular function after two hours of global ischaemia in rat heart. Cardiovasc Res. 1993;27:770-773.

45. Hock CE, Beck LD, Papa LA. Peptide leukotriene receptor antagonism in myocardial ischaemia and reperfusion. Cardiovasc Res. 1992;26:1206-1211.

46. Zhang WP, Wei EQ, Mei RH et al. Neuroprotective effect of ONO-1078, a leukotriene receptor antagonist, on focal cerebral ischemia in rats. Acta Pharmacol Sin. 2002;23:871-877.

47. Fang SH, Wei EQ, Zhou Y et al. Increased expression of cysteinyl leukotriene receptor-1 in the brain mediates neuronal damage and astrogliosis after focal cerebral ischemia in rats. Neuroscience. 2006;140:969-979.

48. Hahn RA, MacDonald BR, Morgan E et al. Evaluation of LY203647 on cardiovascular leukotriene D4 receptors and myocardial reperfusion injury. J Pharmacol Exp Ther. 1992;260:979-989.

49. Hahn RA, MacDonald BR, Simpson PJ et al. Antagonism of leukotriene B4 receptors does not limit canine myocardial infarct size. J Pharmacol Exp Ther. 1990;253:58-66.

50. Adamek A, Jung S, Dienesch C, et al. Role of 5-lipoxygenase in myocardial ischemia-reperfusion injury in mice. EurJ Pharmacol. 2007;571:51-54.

51. Shekher A, Singh M. Role of eicosanoid inhibition of ischemia reperfusion injury: intact and isolated rat heart studies. Methods Find Exp Clin Pharmacol. 1997;19:223-229.

52. Jiang W, Hall SR, Moos MP, Cao RY, Ishii S, Ogunyankin KO, Melo LG, Funk CD. Endothelial cysteinyl leukotriene 2 receptor expression mediates myocardial ischemia-reperfusion injury. Am J Pathol. 2008;172:592-602. 